\title{
A IMPORTÂNCIA DA PREVENÇÃO DE ACIDENTES NA INFÂNCIA: UM RELATO DE EXPERIÊNCIA
}

\section{THE IMPORTANCE OF CHILDHOOD ACCIDENT PREVENTION: A REPORT OF EXPERIENCE}

\section{LA IMPORTANCIA DE LA PREVENCIÓN DE ACCIDENTES EM LA INFANCIA: UM RELATO DE EXPERIENCIA}

\author{
Edficher Margotti ${ }^{1}$, Pedro Paulo Silva da Costa $^{2}$, Allyson Maycon Chaves Corrêa ${ }^{3}$
}

\begin{abstract}
RESUMO
Objetivo: relatar a experiência vivenciada por acadêmicos de enfermagem na realização de ações educativas do projeto de extensão "Acidentes domésticos na infância não é brincadeira" realizado nas enfermarias pediátricas da Fundação Santa Casa de Misericórdia do Pará (FSCMPA) e do Hospital Universitário João de Barros Barreto (HUJBB). Método: trata-se de relato de experiência, vivenciado por acadêmicos de enfermagem, do sexto período, na realização do projeto de extensão apoiado pela Universidade Federal do Pará, executando ações educativas para orientar crianças, pais ou cuidadores, sobre prevenção de acidentes domésticos na infância, utilizando-se do lúdico, em especial do teatro de fantoches. Resultados: foi beneficiado um grupo de 800 pessoas, foram realizados oito encontros, balizados acerca dos temas: afogamento, intoxicação por produtos de limpeza, objetos no nariz e ouvido, queimaduras, quedas, escalpelamento, choque elétrico e atropelamento. Os encontros proporcionaram momentos de bem-estar, valorização da vida e saúde da criança, satisfação, relaxamento e interação. Conclusão: a experiência vivenciada pelos acadêmicos de enfermagem foi de que as ações educativas realizadas pelo projeto, através da atividade lúdica ( teatro de fantoches), foram importante na promoção da saúde e na prevenção de acidentes domésticos na infância.
\end{abstract}

Descritores: Prevenção de Acidentes, Pediatria, Saúde da Criança, Acidentes Domésticos.

\begin{abstract}
Objective: to report the experience of nursing students in carrying out educational actions of the "Domestic accidents in childhood is not a joke" project carried out in the pediatric wards of the Santa Casa de Misericórdia Foundation of Pará (FSCMPA) and the University Hospital João de Barros Barreto (HUJBB). Method: This is an experience report, carried out by nursing academics from the sixth period, in the extension project supported by the Federal University of Pará, carrying out educational actions to guide children, parents or caregivers on the prevention of domestic accidents in Brazil. childhood, using the ludic, especially the puppet theater. Results: a group of 800 people benefited, eight meetings were held on the

\footnotetext{
${ }^{1}$ Enfermeira Obstetra. Doutora em Pediatria e Saúde da Criança, Docente do Semi Internato em Pediatria da Universidade Federal do Pará (UFPA). Belém, PA, Brasil. edficher@ufpa.br.

${ }^{2}$ Graduando, Instituto de Ciências da Saúde, Faculdade de Enfermagem da Universidade Federal do Pará (UFPA). Belém, PA, Brasil. Silvapedro1188@gmail.com;

${ }^{3}$ Graduando, Escola de Enfermagem "Magalhães Barata" - Campus IV/ Centro de Ciências Biológicas e da Saúde (CCBS)/ Universidade Estadual do Pará (UEPA). Belém, PA, Brasil. AllysonMaycon10@ gmail.com
} 
following topics: drowning, intoxication from cleaning products, nose and ear objects, burns, falls, scalping, electric shock and trampling. The meetings provided moments of well-being, appreciation of the child's life and health, satisfaction, relaxation and interaction. Conclusion: the experience of the nursing students was that the educational activities carried out by the project, through the play activity (puppet theater), were important in the promotion of health and in the prevention of childhood domestic accidents.

Descriptors: Accident Prevention, Pediatrics,Child health, Domestic Accidents.

\section{RESUMEN}

Objetivo: relatar la experiencia vivenciada por académicos de enfermería en la realización de acciones educativas del proyecto de extensión "Accidentes domésticos en la infancia no es broma" realizado en las enfermerías pediátricas de la Fundación Santa Casa de Misericordia del Pará (FSCMPA) y del Hospital Universitario João de Barros Barreto (HUJBB). Metodología: se trata de relato de experiencia, vivido por académicos de enfermería, del sexto período, en la realización del proyecto de extensión apoyado por la Universidad Federal de Pará, ejecutando acciones educativas para orientar a niños, padres o cuidadores, sobre prevención de accidentes domésticos en la calle la infancia, utilizando el lúdico, en especial del teatro de títeres. Resultados: se benefició un grupo de 800 personas, se realizaron ocho encuentros, balizados acerca de los temas: ahogamiento, intoxicación por productos de limpieza, objetos en la nariz y el oído, quemaduras, caídas, escalpelamiento, choque eléctrico y atropellamiento. Los encuentros proporcionaron momentos de bienestar, valorización de la vida y salud del niño, satisfacción, relajación e interacción. Conclusión: la experiencia vivenciada por los académicos de enfermería fue que las acciones educativas realizadas por el proyecto, a través de la actividad lúdica (teatro de títeres), fueron importantes en la promoción de la salud y en la prevención de accidentes domésticos en la infancia.

Descriptores: Prevención de Accidentes, Pediatría, Salud del Nino,Accidentes Domésticos.

\section{INTRODUÇÃO}

Quando se convive com crianças, muitos são os acidentes que podem ocorrer no cotidiano; às vezes, nem se imagina que algo possa acontecer, porém é sempre bom estar atendo para as adversidades que uma criança nos reserva. De acordo com a Organização Mundial da Saúde (OMS) acidente é todo o acontecimento independente da vontade humana, não intencional, desastroso e evitável, desencadeado pela ação repentina e rápida, produtora ou não de lesão corporal, no âmbito doméstico ou nos outros ambientes sociais como trânsito, escola, desporto e lazer, provocado por uma força exterior, que age rapidamente e que se manifesta por dano corporal ou mental. Ou seja, os acidentes atualmente são classificados como causas externas. ${ }^{1}$ As crianças, nessa perspectiva, são o grupo mais suscetível aos desastres e acidentes no contexto populacional. Quanto menor e mais imatura for à criança, menor será sua compreensão de risco e maior a vulnerabilidade e dependência de outras pessoas contra acidentes e desastres. ${ }^{1}$

Nas atividades que com elas realizamos, os riscos estão presentes, portanto, é de responsabilidade do 
cuidador atenção especial e conhecimento para que não ocorram descuidos e que sempre uma medida imediata para solucionar o problema esteja disponível em nossas práticas. A vulnerabilidade delas aos acidentes irá variar de acordo com a coordenação de seu sistema nervoso, habilidade motora, senso de percepção de risco e da proteção a ela dispensada pela mãe e demais familiares. ${ }^{2}$

Quanto ao local do acidente infantil, são mais frequentes no interior do domicílio, espaço para o crescimento e desenvolvimento da criança, o qual pode se mostrar hostil em alguns momentos. Isso ocorre porque é o local de maior permanência da criança. ${ }^{3}$

No Brasil, um estudo realizado com 7.123 crianças de idade inferior a 10 anos, evidenciou que $96,8 \%$ foram vítimas de acidentes, a maioria das ocorrências $(66,6 \%)$ incidiu no domicílio, tendo o corte como lesão mais frequente $(35,7 \%)$. Além disso, do total de crianças, $18,9 \%$ tinham até um ano de idade e $41,6 \%$ de dois a cinco anos. 4

Nessa perspectiva, as consequências dos acidentes repercutem-se não só na saúde física das crianças, como também no seu bem-estar e das famílias; causam na sociedade custos altíssimos em tratamentos, assistência, dependendo da gravidade, para toda a vida. Assim, o acidente doméstico foi caracterizado como uma das principais causas de assistência, internações, incapacidades e mortes na infância, e tem contribuído consideravelmente para a alta taxa de morbidade e mortalidade infantil. ${ }^{5}$

$\mathrm{O}$ que justificou nosso trabalho foi a observação da alta incidência de internações nas unidades pediátricas, motivados por acidentes domésticos ocorridos no próprio domicílio da criança, e a necessidade urgente em diminuir essa incidência de internações por esse motivo, surgindo com isso a necessidade premente em se fazer e executar programas.

$\mathrm{O}$ artigo tem o objetivo de relatar a experiência vivenciada por acadêmicos de enfermagem na realização de ações educativas do projeto de extensão, intitulado "Acidentes domésticos na infância não é brincadeira", realizado nas enfermarias pediátricas da Fundação Santa Casa de Misericórdia do Pará (FSCMPA) e do Hospital Universitário João de Barros Barreto (HUJBB), executando ações educativas para orientar crianças, pais, cuidadores e responsáveis, sobre prevenção de acidentes domésticos na infância, utilizando-se do lúdico, em especial do teatro de fantoches.

\section{MÉTODO}

Trata-se de um relato de experiência embasado na vivência prática por acadêmicos de enfermagem a partir do 
desenvolvimento do Projeto de Extensão intitulado "Acidentes domésticos na infância não é brincadeira”. Esse método foi escolhido por se tratar de uma ferramenta descritiva, que gera uma reflexão sobre ações que estiveram presentes em uma situação vivenciada no âmbito profissional de interesse para a academia científica. ${ }^{6}$

Realizado no período de março a junho de 2017, nas Enfermarias Pediátricas da Fundação Santa Casa de Misericórdia do Pará (FSCMPA) e do Hospital Universitário João de Barros Barreto (HUJBB); tem como público alvo crianças com faixa etária dos 5 a 10 anos de idade e pais, responsáveis ou cuidadores das crianças hospitalizadas. O projeto contou com 800 pessoas, dentre elas crianças hospitalizadas, pais, responsáveis e/ou cuidadores. A amostragem não foi probabilística, e sim de voluntários.

Para a execução do projeto junto aos hospitais, houve o contato prévio com a coordenação da instituição e da enfermagem, objetivando o delineamento e a organização do cronograma para o início das atividades.

Em um primeiro momento, fez-se um levantamento quanto ao número de crianças hospitalizadas por acidentes domésticos, o recurso utilizado foi o censo diário da unidade, que contém essa informação, em seguida o instrumento para a coleta dos dados foi um questionário simples distribuído aos pais, responsáveis ou acompanhantes, sobre o conhecimento dos principais acidentes domésticos e pedindo sugestões de temas relacionados a acidentes domésticos na infância para serem abordadas nos dias das ações.

O intuito foi de observar o ímpeto das dinâmicas, demonstrado para as crianças e pais, responsáveis ou cuidadores, quanto a relevância da temática: acidentes domésticos na infância. Tema esse abordado nas ações educativas de forma lúdica e interativa, possibilitando a troca de conhecimento e experiências.

As crianças, pais, responsáveis ou cuidadores, eram convidados a sentarem, para melhor visualizar a apresentação do teatro e para um maior conforto. As peças teatrais incluíam 5 personagens: Seu João, Dona Ana, Marcelinha, Maria e a Enfermeira Joana.

Após o término de cada sessão teatral, os personagens, faziam questionamentos aos participantes e esclareciam todas as dúvidas vindas das crianças, pais e responsáveis. Os adultos respondiam a algumas perguntas, como por exemplo, se achavam importante aquele assunto, se entenderam o que foi abordado, o que fazer no caso de ocorrer aquele tipo de acidente dentro de casa e se tinham alguma dúvida. Ressalta-se que após cada encontro era realizada uma avaliação do 
projeto junto aos pais e responsáveis, oportunizando que cada participante pudesse destacar pontos positivos e outros a serem melhorados. Para avaliar o projeto e a satisfação do público em relação à ação de promoção da saúde, foi criado um instrumento avaliativo, que era preenchido após cada atividade.

Este projeto e consequentemente os dados aqui mostrados, foram extraídos do projeto de extensão, denominado "Acidentes domésticos na infância não é brincadeira" que foi financiado pela PROEX no Edital PIBEX 2017, da Universidade Federal do Pará (UFPA). Não necessitou da aprovação do CEP, mas foi aceito e aprovado pelos hospitais.

\section{RESULTADOS}

Foram beneficiados com as atividades lúdicas um grupo de mais de 800 crianças, pais, responsáveis e/ou cuidadores. Foram realizadas atividades lúdicas, utilizando se o teatro de fantoches. Foram realizados oito peças teatrais. A cada encontro, era abordado um acidente doméstico diferente, como afogamento, intoxicação por produtos de limpeza, objetos no nariz e ouvido, queimaduras, quedas, escalpelamento, choque elétrico e atropelamento.

A recepção das crianças, pais, responsáveis e/ou cuidadores em ambos os hospitais foi positiva, uma vez que $100 \%$ responderam ser importante a abordagem do tema, dos quais $40,5 \%$ classificaram a intervenção como excelente, $30 \%$ como ótima e $29,5 \%$ como boa, uma vez que muitas crianças que ali estavam, eram por motivos de acidentes ocorridos dentro de suas próprias casas.

Ao iniciar as atividades nas pediatrias dos hospitais, nos primeiros encontros com as crianças, elas se mostraram tímidas. Durante a realização do teatro de fantoches, foi notório o interesse de todas, demonstrado por meio de gestos faciais e corporais, satisfação, contentamento e interação com os bonecos. A escolha pela brincadeira popular lúdica e assuntos do cotidiano relacionado ao acidente doméstico, facilitaram a aceitação por parte das crianças e consequentemente potencializaram a construção do conhecimento acerca das principais causas de acidentes com crianças que ocorrem no domicílio e como preveni-las.

Foi observado que os pais, responsáveis e cuidadores, apresentam uma certa carência de informações relacionados ao tema. Os adultos possuíam um déficit de conhecimento relacionado aos acidentes domésticos envolvendo crianças, fato evidenciado pelo grande número de questionamentos feitos aos acadêmicos. 
Verificou-se, também, que os acadêmicos de enfermagem ganharam um empoderamento, receberam algum tipo de contentamento, entusiasmo e prazer, uma vez que se explicou à importância da prevenção de acidentes na infância. Ficou evidente no trabalho que é necessário que haja preparação do enfermeiro educador para identificação das fontes que oferecem riscos à criança. Foi de suma importância à reflexão do grupo de acadêmicos para responder as perguntas advindas das crianças, dos pais, responsáveis ou dos cuidadores.

\section{DISCUSSÃO}

Vale ressaltar que houve a promoção do tema, o desenvolvimento e a transferência de conhecimento, principalmente com as perguntas feitas e respondidas pelas crianças. A percepção das crianças em relação à atividade realizada, compreendida mediante indagações, apresentou-se de maneira extremamente positiva, tanto para o objetivo da atividade, quanto para a formação dos alunos a partir de uma percepção mais humanística. Outros trabalhos também mostraram a educação continuada em saúde, percebida positivamente por escolares $^{6}$ e por adolescentes. $^{3}$
As temáticas discutidas nesse projeto foram pertinentes e a utilização da metodologia lúdica, com ênfase no diálogo, mostrou-se adequada em relação aos participantes, permitindo a reflexão, a troca de experiências e a construção do aprendizado conjunto. No que tange à percepção sobre a utilização do lúdico para atividades de promoção à saúde junto às crianças, os ouvintes compreenderam que este tipo de estratégia configurou-se como adequada e eficiente. Outro projeto também perpassou pela questão da formação individual, mostrando que a abordagem lúdica representar um importante instrumento de auxílio para o processo de formação da conscientização das crianças. ${ }^{7}$

A brincadeira é uma atividade que as crianças gostam e que é necessária para sua vida, pois, assim como o ato de comer ou dormir, a brincadeira exerce uma função orgânica e psicossocial no desenvolvimento da criança. Também, é por meio da brincadeira que a criança constrói conhecimento sobre o mundo e sobre si mesmo. ${ }^{7}$ É concordando com esse princípio que nossas atividades incluem o "teatro de fantoches", que além de proporcionar o lazer, o divertimento e o sentimento de satisfação, contribuem para o desenvolvimento intelectual, psicológico e social das crianças hospitalizadas. 
As brincadeiras imprimem características que permitem que os participantes, expressem, suas impressões, conceitos, opiniões e concepções sobre o tema proposto, assim como permite trabalhar reflexivamente as manifestações apresentadas pelo grupo a respeito do tema abordado. $^{8}$ Concordando com isso, introduzimos a ludicidade e ampliamos as oportunidades educacionais para o além do processo de aprendizagem. Isso não deixa, também, de ser um fenômeno cultural, uma vez que se constitui em um conjunto de conhecimentos, sentidos e significados construídos pelos sujeitos nos contextos históricos e sociais em que se inserem.

Os profissionais que lidam com o público infantil devem se atentar quanto às características da criança, pois isso pode facilitar a ocorrência de determinados tipos de acidentes, contribuindo para que a prevenção seja mais eficaz. Nessa perspectiva, especialmente com relação ao acidente infantil, é notória que a eficácia de qualquer estratégia dependerá da parceria de trabalho em conjunto entre as famílias das crianças, escolas e profissionais de saúde.

É importante e necessário que sejam realizadas atualizações do profissional enfermeiro, com a tentativa de esclarecer as dúvidas e conhecer o público infantil. ${ }^{9}$ Concordando com isso; é que o nosso projeto introduziu o brinquedo, em especial o fantoche, na prática assistencial de enfermagem, na tentativa de minimizar o número de acidentes domésticos envolvendo crianças.

Autores refletem sobre o exercício da prática educativa do enfermeiro à criança e mostram a importância de observar o contexto em que a criança vive, com a finalidade de proporcionar um cuidado integral e individualizado. ${ }^{10}$ Nessa mesma perspectiva, nosso trabalho e nossos acadêmicos de enfermagem tentam fazer com que a redução dos acidentes possam ser alcançadas mediante prevenções educativas com pais, responsáveis ou cuidadores, assegurando informações e procedimentos necessários para proteger a criança em relação ao acidente.

É importante ser levado em consideração a necessidade de práticas lúdicas educativas na vida. Assim, é necessário que o transmissor da informação insira o brincar em um projeto educativo e preventivo, ou seja, ter a consciência da importância de sua ação em relação à aprendizagem. ${ }^{11}$ É de comum acordo com esse trabalho, que os acadêmicos de enfermagem fazem da transmissão de informações neste contexto ser de estrema importância, e a educação continuada através do lúdico, ser apresentada como um instrumento indispensável. O profissional enfermeiro é apto a desenvolver programas para 
prevenção e atividades educativas para o autocuidado, sendo portanto, de extrema importância para a redução de tais acidentes na sociedade.

O presente estudo traz contribuições significativas para a saúde, considerando que as atividades de promoção à saúde da criança influenciam diretamente na satisfação e produtividade dos pais, ou responsáveis. Este estudo apresenta um modelo de ação de fácil acesso e realização, que mostrou resultados satisfatórios com a equipe de enfermagem, sendo relevante para ampliação dessa rotina em outros serviços.

\section{CONCLUSÃO}

Concluímos que a experiência vivenciada pelos acadêmicos de enfermagem nas enfermarias pediátricas da FSCMPA e do HUJBB, foi de que as ações educativas realizadas pelo projeto, através da atividade lúdica ( teatro de fantoches), foram importante na promoção da saúde e na prevenção de acidentes domésticos na infância.

A educação continuada e a conscientização, utilizando fantoches e abordando o tema acidentes domésticos na infância foram alcançados, todavia ainda há muito no que trabalhar, pois a educação é algo que é implantada lentamente. Desta forma, cabe aos pais, responsáveis e cuidadores das crianças, a busca de aprimoramento de seus conhecimentos, para a melhoria do cuidado a criança, proporcionando, assim, maior segurança física para a mesma.

Podemos pensar em como os brinquedos, nesse caso os fantoches, são instrumentos que deveriam ser levados para o ambiente hospitalar com mais frequência. Socializar o conhecimento com a educação e utilizar esse instrumento é muito importante: não só resgata a ludicidade, mas como também mostra a valorização da prática no seu aspecto educacional.

Evidencia-se algumas limitações para o desenvolvimento das atividades: a escolha pelas crianças que não tinham dificuldade na locomoção, dificultou a participação da maioria das crianças e pais; o curto período de tempo (um ano) para realização do projeto, visto que existe uma alta demanda nas internações por motivos de acidentes domésticos infantis; a realização do trabalho em apenas dois hospitais, haja visto que existem muitos hospitais na cidade.

\section{REFERÊNCIAS}

1. Organização Mundial da Saúde. Cid-10: classificação estatística internacional de doenças e problemas relacionados à saúde. 8ed. São Paulo: EDUSP; 2000.

2. Gomes LMX, Rocha RM, Barbosa TLA, Silva CSO. Descrição dos acidentes domésticos ocorridos na 
infância. Mundo Saúde. 2013; 37(4); 394-400.

3. Santos SJ, Andrade RD, Mello DF, Maia MAC. Educação em saúde na adolescência: contribuições da estratégia saúde da família. Rev Soc Bras Enferm Pediatras. jul 2014; 14(1):20-6.

4. Bezerra MAR, Rocha RC, Negreiros FS, Lira FMOM, Sousa LT, Santiago SCG. Acidentes domésticos em crianças: concepções práticas dos agentes comunitários de saúde. Cogitare Enferm. 2014; 19(4):716-24.

5. Ministério da Saúde (Brasil). Portaria $\mathrm{n}^{\circ}$ 737 de 16 de maio de 2001. Política nacional de redução da morbimortalidade por acidentes e violências [Internet]. D.O.U., Brasília, DF: Ministério da Saúde; 2001 [citado em 12 jul 2017]. Disponivel em: http://bvsms.saude.gov.br/bvs/saudelegi s/gm/2001/prt0737_16_05_2001.html

6. Leite CT, Vieira RP, Machado CA, Quirino GS, Machado MFAS. Prática de educação em saúde percebida por escolares. Cogitare Enferm. jan/mar 2014; 19(1):13-19.

7. Soares, FM, Gonçalves ABC, Alvim RO, Júnior CAM, Oliveira CM. Conscientização infantil: abordagem lúdica sobre utilização de recursos naturais. Rev Ciênc Ext. 2017; 13(3):87-92.

8. Melo MCH, Cruz G. Roda de conversa: uma proposta metodológica para a construção de um espaço de diálogo no ensino médio. Imagens Educ. 2014; 2(4):31-39.

9. Gomes MFP, Silva ID, Capellini VK. Conhecimento dos profissionais de enfermagem sobre a utilização do brinquedo no cuidado as crianças hospitalizadas. Rev Enferm UFPI. jan/mar 2016; 5(1):23-27.

10. Rocha GST, Araújo Filho ACA, Nunes BMVT, Rocha SS. Prática educativa do enfermeiro na consulta de enfermagem à criança na perspectiva de
Madeleine Leininger. Rev Enferm UFPI. abr/jun 2015; 4(2):124-9.

11. Mendes LC, Elias TC, Santos TN, Tayar EM, Riul SS. Atividades educativas estimulando o autocuidado e prevenção do câncer feminino. Rev Enferm Atenção Saúde [Internet]. jan/jun 2017 [citado em 02 jul 2017]; 6(1):140-7. Disponível em: http://seer.uftm.edu.br/revistaeletronica/ index.php/enfer/article/view/1792/pdf

RECEBIDO: $16 / 08 / 2017$

APROVADO: $18 / 07 / 2018$

PUBLICADO: 07/2018 